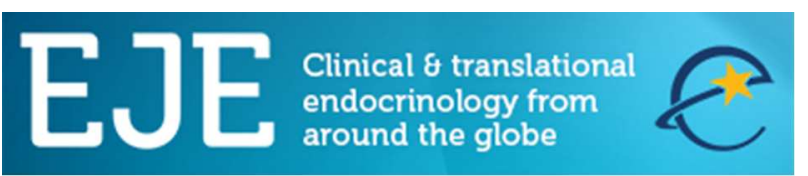

\title{
Occurrence of malignant neoplasia in patients with primary hyperparathyroidism
}

\begin{tabular}{|c|c|}
\hline Journal: & European Journal of Endocrinology \\
\hline Manuscript ID & EJE-17-0028 \\
\hline mstype: & Clinical Study \\
\hline Date Submitted by the Author: & 13-Jan-2017 \\
\hline Complete List of Authors: & $\begin{array}{l}\text { Palmieri, Serena; Ospedale Maggiore Policlinico, Unit of Endocrinology and } \\
\text { Metabolic Diseases; Universita degli Studi di Milano, Department of Clinical } \\
\text { Sciences and Community Health } \\
\text { Roggero, Letizia; Universita degli Studi di Milano, Dpt. of Medical Sciences } \\
\text { and Community Health; Ospedale Maggiore Policlinico, Unit of } \\
\text { Endocrinology and Metabolic Diseases } \\
\text { Cairoli, Elisa; Universita degli Studi di Milano, Dpt. of Medical Sciences and } \\
\text { Community Health; Ospedale Maggiore Policlinico, Unit of Endocrinology } \\
\text { and Metabolic Diseases } \\
\text { Morelli, Valentina; Universita degli Studi di Milano, Dpt. of Medical Sciences } \\
\text { and Community Health; Ospedale Maggiore Policlinico, Unit of } \\
\text { Endocrinology and Metabolic Diseases } \\
\text { Scillitani, Alfredo; Casa Sollievo della Sofferenza Scientific Institute, Unit of } \\
\text { Endocrinology } \\
\text { Chiodini, Iacopo; Ospedale Maggiore Policlinico, Unit of Endocrinology and } \\
\text { Metabolic Diseases } \\
\text { Eller-Vainicher, Cristina; Ospedale Maggiore Policlinico, Unit of } \\
\text { Endocrinology and Metabolic Diseases }\end{array}$ \\
\hline Keywords: & Primary hyperparathyroidism, malignancy, breast cancer, vitamin D \\
\hline
\end{tabular}

\section{SCHOLARONE ${ }^{\text {MN }}$}

Manuscripts 
4

$5 \quad{ }^{1}$ Department of Medical Sciences and Community Health, University of Milan, Milan, Italy.

$6{ }^{2}$ Unit of Endocrinology and Metabolic Diseases, IRCCS Cà Granda-Ospedale Maggiore Policlinico Milan,

7 Italy. ${ }^{3}$ Unit of Endocrinology, "Casa Sollievo della Sofferenza”, Hospital, IRCCS, San Giovanni Rotondo,

8 Foggia, Italy.

9 Short title: Cancer and primary hyperparathyroidism

10 Keywords: Primary hyperparathyroidism, malignancy, breast cancer, vitamin D

11 Word count: 3284

12 Number of figures/tables: $2 / 3$

13 Disclosure Summary: Authors have nothing to disclose.

14 Corresponding author and person to whom the reprint request should be addressed:

15 Iacopo Chiodini, MD

16 Unit of Endocrinology and Metabolic Diseases, Padiglione Granelli,

17 Fondazione IRCCS Cà-Granda, Ospedale Maggiore Policlinico

18 Via Francesco Sforza 35, 20122 Milan, Italy.

19 Phone : +39-2-55033355; Fax : +39-2-50320605; e-mail: iacopo.chiodini@unimi.it 


\section{Abstract}

Background. The association between primary hyperparathyroidism (1HPT) and cancer is debated. The present study was aimed to investigate the occurrence of neoplasia in 1HPT.

Methods: All consecutive patients ( $\mathrm{n}=1750)$ referred to our "Osteoporosis and Metabolic Disease" outpatients clinic for osteoporosis or hypercalcemia were eligible for the study. The exclusion criteria were: the finding of osteoporosis and/or altered calcium-phosphorous metabolism in the context of investigations for malignancy, the presence of diseases known to influence the cancer risk and the heavy smoking habit. Eventually, 1606 patients (1407 females, 199 males) were enrolled. In all patients calcium-phosphorous metabolism, PTH and vitamin D levels were measured and the occurrence of cancer during the 10 years prior the study inclusion was recorded.

Results: 163 patients had 1HPT while 1443 had not. Patients with and without 1HPT were comparable for

31 age and gender. In 1HPT patients the occurrence of all, breast, kidney and skin cancer was significantly higher $(21.5 \%, 12.2 \%, 2.5 \%, 1.8 \%$, respectively) than in patients without $1 \mathrm{HPT}(12.4 \%, 6.9 \%, 0.3 \%, 0.3 \%$, $\mathrm{p}<0.05$ for all comparisons). The 1HPT presence was significantly associated with the occurrence of all neoplasia and of breast, skin and kidney neoplasia (odds ratio, 95\% confidence interval, P value: 1.93, 1.27$2.92,0.002 ; 1.93,1.11-3.35,0.002 ; 9.18,2.16-38.8,0.003 ; 8.23,1.71-39.5,0.008$, respectively), after adjusting for age, gender (as appropriate), smoking habit and vitamin D levels. neoplasia is increased. 


\section{Introduction}

Primary hyperparathyroidism (1HPT) affects at least 1 in 1000 persons and its incidence rises with age, with a peak in the seventh decade $(1,2)$. The 1 HPT risk is 5 -fold higher in women than in men after 75 years of age, while it is comparable between sexes before 45 years of age (1). In the majority (i.e. 80-85\%) of cases $1 \mathrm{HPT}$ is due to a single gland adenoma, with hyperplasia and parathyroid carcinoma accounting for the $10-15 \%$ and $<1 \%$ of cases, respectively (2).

The 1HPT condition is characterized by hypercalcemia, increased urinary calcium excretion and inappropriately high or unsuppressed levels parathyroid hormone (3), and it may lead to osteoporosis, fragility fractures and kidney stones and, possibly, to an increased risk of cardiovascular events and neuropsychological alterations (4). Besides these consequences, given the evidence of an increased cancer-related mortality in 1HPT $(5,6)$ and of the possible anti-apoptotic action of the parathyroid hormone (PTH) (7-9), in the past years some authors investigated the possibility of an increased risk of malignancies in this condition $(5,6,10-18)$. Interestingly, cancer is now a known feature of chronic kidney disease, which is characterized persistently elevated PTH values (19).

However, while some studies found an increased risk of some malignancies, such as breast, skin, colon, thyroid and kidney cancer (10-16), other investigations were not able to find any association between 1HPT and malignant neoplasia $(17,18)$. These discordances are probably due to the different designs (i.e. retrospective or prospective), different settings (i.e. national registers- or population-based) and different inclusion criteria (i.e. all patients diagnosed with $1 \mathrm{HPT}$ or only surgically treated 1HPT patients) of the available studies. In addition, given that many studies were based on National Registers data, the origin of the 1HPT diagnosis was not reported. This is of utmost importance, since 1HPT is often asymptomatic and its diagnosis is frequently made in the context of routine exams. Therefore, it is not possible to exclude that the increased 1HPT prevalence, that has been described among patients with cancer in some studies, could have been related, in fact, to the increased number of biochemical investigations routinely performed in patients with neoplasia. Finally, a complete characterization of 1HPT patients regarding their disease activity, comorbidities and risk factors for cancer was lacking in all available studies, rendering the results scarcely comparable. 
In order to overcome these pitfalls, we designed a study for comparing the occurrence of malignant neoplasia in patients referred to our "Osteoporosis and Metabolic Disease" outpatient clinic and subsequently diagnosed with or without 1HPT.

\section{Design of the study, patients and methods}

Design of the Study and patients

All Caucasian patients ( $\mathrm{n}=1750,1532$ females, 218 males) referred to the "Osteoporosis and Metabolic Disease" tertiary care outpatient clinic since May $1^{\text {st }}, 2014$ to May $31^{\text {st }} 2016$ at Fondazione IRCCS Cà Granda-Ospedale Maggiore Policlinico in Milan (Italy), for the first report of osteoporosis and/or fragility fracture and/or increased calcium and/or PTH levels were considered eligible for the study. Since patients affected with osteoporosis may be referred to our outpatient clinic (a tertiary care center for the study of hyperparathyroidism and severe osteoporosis) even for the skeletal consequences of systemic diseases (20), some of which could per se influence the cancer risk, the study protocol was designed in order to avoid the foreseeable potential selection bias. Therefore it comprised the following exclusion criteria: I) the finding of osteoporosis and/or fragility fracture and/or increased calcium and/or PTH levels in the context of investigations for the staging or follow-up of malignancy; II) the patients' addressing for the need of the prescription of a bone-active drug in the context of aromatase inhibitors therapy, as recommended by our National Guidelines (21); III) the presence of neoplasia in the context of a previously identified familiar and/or hereditary syndrome (i.e. multiple endocrine neoplasia, hereditary breast or ovarian cancer) (22); IV) the presence of diabetes, obesity (i.e body mass index, BMI, $\geq 30 \mathrm{~kg} / \mathrm{m} 2$ ), chronic liver diseases or other chronic disorders (i.e renal and hepatic transplant, chronic inflammation diseases) and/or substances and therapies (i.e glucocorticoids, immunosuppressants, alcohol abuse, external radiation) known to increase the cancer risk and/or calcium-phosphorous metabolism (23); V) the presence of heavy smoking habit ( $\geq 1$ pack/day) (24). On the basis of these criteria, 144 patients ( $n=6, n=6, n=20, n=97$ and $n=15$ fulfilling the criteria I, II, III, IV and V, respectively) did not entry the study, and eventually 1606 patients (1407 females, 199 males) were enrolled (Figure 1)

From all patients, the occurrence of malignant neoplasia during the 10 years prior the study inclusion was recorded. The diagnosis of cancer have been ascertained by verifying the medical reports. This length of 
94 observational period of time has been decided since in 1HPT patients the diagnostic delay may be even of 10

years (25). In addition, from all patients we obtained information regarding the occurrence of fragility fractures (at spine, ribs, wrist, hip, and proximal humerus), cardiovascular events (i.e. myocardial infarction, stroke, transient ischemic attack, angina pectoris, pulmonary embolism, intracerebral hemorrhage, peripheral artery disease), hypertension and dyslipidemia during the 10 years prior the study inclusion. In all patients the diagnosis of osteoporosis or osteopenia was done on the basis of a dual X-ray absorptiometry (DXA) bone mineral density (BMD) assessment and/or of the occurrence of a fragility fracture $(26,27)$. Subjects were considered current smokers if they smoked more than 5 cigarettes/day and/or 10 packs/year (28).

All patients underwent the biochemical testing for osteoporosis suggested by both the protocol approved in our Centre (20) and our national Guidelines (29), including, in particular, serum calcium, albumin, phosphorous, creatinine, PTH, 25hydroxy-cholecalciferol (25OHVitD), 24-h urine calcium and creatinine excretion levels. All patients with increased albumin-adjusted serum calcium levels, confirmed at least twice, were tested for ionized serum calcium levels. Primary hyperparathyroidism was diagnosed in the presence of hypercalcemia and elevated or inappropriately normal PTH levels, after the evaluation of calcium clearance:creatinine clearance ratio, in order to rule out familial hypocalciuric hypercalcemia (30). Eventually 163 patients (148 females, 15 males) were found to be affected with 1HPT while 1443 (1259 females and 184 males) were not and were used as control group.

We reviewed the medical records preceding the study entry of all 1HPT patients. In 40 patients (37 females, 3 male, age $70.7 \pm 11.3$ years) we found that the hypercalcemia was already present at least 5 years before the enrolment. In this subgroup of 1HPT patients we assessed the occurrence of malignancies during the period of time between the first finding of hypercalcemia and the study entry. These data were compared with those of 120 age-, and gender-matched subjects without 1HPT, who had been randomly selected from the whole group of control subjects.

The study complies with the Declaration of Helsinki and it has been approved by the Ethical Committee of Fondazione IRCCS Cà Granda-Ospedale Maggiore Policlinico, Milan, Italy. An informed consent has been obtained from each patient. 
122

123

124

125

126

127

128

129

130

131

132

133

134

135

136

137

138

139

140

141

142

143

144

145

146

147 25OHVitD levels and smoking habit.

148 Values of $\mathrm{p}<0.05$ were considered significant.

\section{Methods} respectively (normal levels above $30 \mathrm{ng} / \mathrm{mL}$ ). previously described by Genant and coauthors. (32).

\section{Statistical analysis} variables were compared by $\chi^{2}$ test.

Serum and urinary calcium, albumin, and phosphate were measured by standard colorimetric techniques. Total calcium was corrected for serum albumin according to the formula: total calcium $(\mathrm{mg} / \mathrm{dL})+(4.4-\text { albumin }(\mathrm{mg} / \mathrm{dL}))^{*} 0.8(31)($ reference interval $8.4-10.2 \mathrm{mg} / \mathrm{dl})$. Serum intact PTH levels were measured by a two-step automated sandwich chemiluminescent immunoassay (DiaSorin), with intra- and interassay coefficients of variation $<10 \%$ (reference interval $6.5-36.8 \mathrm{pg} / \mathrm{mL}$ ). The serum $25 \mathrm{OHVitD}$ concentration was measured by radioimmunoassay (Diasorin), with intra and interassay of $7.2 \%$ and $<12 \%$,

Data regarding BMD were obtained by DXA scans at the spine and hip, performed at our Hospital (Hologic Discovery, Waltham MA, USA) at lumbar spine (LS; in vivo precision 1.0\%), total femur (FT, in vivo precision $1.7 \%$ ) and femoral neck (FN, in vivo precision $1.8 \%$ ) in the $77 \%$ of patients and at other centers (Hologic Discovery and Lunar GE) in the $23 \%$ of patients. Conventional spinal radiographs in lateral and anteroposterior projection (T4-L4) were obtained at baseline in all subjects with standardized technique. Vertebral fractures were diagnosed on visual inspection using the semiquantitative visual assessment

Statistical analysis was performed by SPSS version 21.0 statistical package (SPSS, Inc.). The normality of distribution was checked by Kolmogorov-Smirnov test. The results are expressed as mean \pm standard deviation if not differently specified. The comparison of continuous variables between patients with and without 1HPT was performed using Student's t-test or Mann-Whitney U test, as appropriate. The categorical

The logistic regression analysis assessed the association between the 1HPT diagnosis and the presence of all neoplasia and of the neoplasia that were significantly more frequent in 1HPT patients than in controls, after adjusting for variables that might influence the cancer risk, such as, age, gender (as appropriate), low 
149

150

151

152

153

154

155

156

157

158

159

160

161

162

163

164

165

166

167

168

169

170

171

172

173

174

175

176

\section{Results}

The clinical characteristics of the whole sample of patients $(n=1606)$ are reported in table 1. During the 10 years prior the study entry, a malignant neoplasia occurred in 412 (26.2\%) patients, with the breast, prostate and thyroid cancers being the most frequent ones. The other cancers occurred at colon, uterus, lung, kidney, skin, ovary, liver and pancreas in decreasing order. Breast and uterus cancer were reported only in female subjects.

A condition of 1HPT was found in 163 patients, while 1443 patients were not affected with 1HPT (Figure 1). Among these latter, 47 patients showed a secondary hyperparathyroidism (i.e. elevated PTH in the presence of frankly normal calcium levels) due to hypovitaminosis D. The comparisons between the clinical characteristics of patients with and without $1 \mathrm{HPT}$ are reported in table 2. Patients with and without 1HPT were comparable as far as age, gender, prevalence of hypertension, cardiovascular events, dyslipidemia and osteopenia or osteoporosis was concerned. The fragility fracture prevalence and the smokers were significantly more prevalent among patients without 1HPT. As expected, in 1HPT patients serum and urinary calcium, total alkaline phosphatase and PTH levels were higher, while phosphorous and 25OHVitD lower, than in patients without 1HPT. In 1HPT patients the overall occurrence of cancer and the occurrence of breast, kidney and skin cancer was significantly higher (1.7-, 1.8-, 8.3- and 6-fold increased, respectively) than in patients without 1 HPT. For the other types of cancer a statistically significant difference was not found.

The period of time between the study entry and the occurrence of neoplasia was lower in patients with 1HPT (56.3 \pm 23.1 months, range 36-116 months) than in those without 1 HPT ( $89.0 \pm 33.6$ months, range 24-120 months). The prevalence of subjects in whom the neoplasia occurred at least 5 years before the study entry was higher in 1 HPT patients $(26 / 35,74.3 \%)$ than in controls $(40 / 179,22.3 \%, \mathrm{p}<0.0001)$. In the 40 patients with 1HPT, in whom the hypercalcemia was already evident within 5 years before the study entry, the occurrence of neoplasia during this period of time tended to be higher (9/40 cases, $22.5 \%)$ than that in the 120 subjects without 1 HPT randomly selected as controls ( $14 / 120$ cases, $11.7 \%, \mathrm{p}=0.06)$.

The logistic regression analysis showed that the occurrence of all neoplasia and of breast, skin and kidney neoplasia was significantly associated with the condition of 1HPT even after adjusting for age, gender (only for all cancers and skin cancer), smoking habit and hypovitaminosis D (table 3). Finally, in the 
17747 patients with secondary hyperparathyroidism, the prevalence of all cancers was comparable with that of 178 patients with normal PTH levels (12.8\% vs 12.4\%) and reduced as compared with 1HPT patients (12.8\% vs $17921.5 \%, \mathrm{p}=0.005)$. 
180

181

182

183

184

185

186

187

188

189

190

191

193

194

195

196

197

198

199

200

201

202

203

204

205

206

207

\section{Discussion}

The present study shows that the occurrence of cancer, and in particular of breast, skin and kidney cancer is increased during the 10 years before the diagnosis of $1 \mathrm{HPT}$ even after adjusting for possible confounders such as age, gender, presence of hypovitaminosis D and smoking habit.

The previous studies investigating the occurrence of neoplastic diseases in 1HPT gave discordant results. Indeed, while some data suggested that the 1HPT condition could be associated with an increased risk of breast, skin, colon, thyroid and kidney cancer (10-16), others did not $(17,18)$. The limits of these investigations were related to their retrospective design and to the fact that the origin of the 1HPT and cancer diagnoses was not clearly reported. Therefore, the 1HPT diagnosis could have been done in the context of cancer staging or, viceversa, cancer diagnosis in the context of 1HPT workup. To overcome these pitfalls, in some of these studies the neoplasia that had occurred before the 1HPT diagnosis were excluded $(11,13)$.

This, however, could have introduced a further bias, since in 1HPT patients the diagnostic delay may be even of 10 years (25). Finally, in all previous studies a complete characterization of subjects with and without 1HPT regarding their comorbidities and risk factors for cancer was lacking, rendering the finding of an increased cancer risk in 1HPT not clearly demonstrated.

The design of the present study consented us to overcome most of these pitfalls. Indeed, the inclusion criteria (i.e. the referral to our "Osteoporosis and Metabolic Disease" outpatient clinic) have been the same for patients with and without 1HPT. By excluding patients with the finding of osteoporosis and/or fragility fracture and/or increased calcium and/or PTH levels in the context of investigations for the staging or follow-up of malignancy, we avoided the risk that an increased 1HPT finding in patients with cancer could have been due to the increased number of exams performed in these patients. Similarly, the exclusion of patients with heavy smoking habit, diabetes, obesity and chronic disorders and/or therapies known to increase the cancer risk consented to avoid that a different occurrence of cancer in patients with and without 1HPT could be related to the different healthy status of the subjects included in the study.

In the present study the independent association between the all cancer occurrence and the female gender is probably related to high frequency of breast cancer. The high prevalence of $1 \mathrm{HPT}$ patients and the increased prevalence of smokers and of fractured patients among patients without $1 \mathrm{HPT}$ is not surprising. Indeed, in our tertiary care outpatient clinic patients are referred by their general practitioners generally for 
an inadequate response to a bone-active drug and/or for the occurrence of a fragility fracture, which are both associated with the smoking habit (33), or for high calcium and/or PTH levels, while rarely for densitometric osteoporosis as unique reason. On the other hand, the finding that the prevalence of hypertension, dyslipidemia and cardiovascular events was comparable between patients with and without 1HPT somewhat confirms that the healthy status was similar between the two groups of patients. Therefore, in the absence of these possible confounding factors, the present study strongly suggests that the condition of 1HPT is associated with an increased occurrence of cancer, and, in particular, of breast, skin and kidney cancer, during the 10 years before the 1HPT diagnosis.

It is evident, however, that the cross-sectional design of the present study does not consent to draw conclusions about causality. In addition, we have no certain information about the temporal distance between the onset of the parathyroid disease and the appearance of the malignancy. However, in 1HPT patients the period of time between the diagnosis of cancer and of 1HPT was lower than in subjects without 1HPT and, at variance with these latter, in the majority of $1 \mathrm{HPT}$ patients the neoplasia occurred less than five years before the 1HPT diagnosis (Figure 2). Importantly, in the subgroup of 1HPT patients, in whom the hypercalcemia was already evident at least 5 years before the study entry, the occurrence of neoplasia during this period of time tended to be higher than in controls. Overall these findings are in keeping with the hypothesis of a relationship between the cancer and the $1 \mathrm{HPT}$ occurrence. the cancer risk may persist even after the recovery from 1HPT is against the hypothesis of a causative role of the PTH excess in the cancer pathogenesis (13). In keeping, in the present study we found that patients with secondary hyperparathyroidism (i.e. with increased PTH levels together with normal calcium levels) did not show an increased occurrence of neoplasia as compared with patients with normal PTH and calcium levels.

230 Even if we have no information regarding the length of the secondary hyperparathyroidism, that could have 231 influenced the results, this finding is in keeping with a previous study suggesting that patients with secondary hyperparathyroidism had an insignificant overall cancer risk (11). In addition, a previous study suggested a

233 possible role of the increased calcium level, in the breast cancer development (34). Overall, these data

234 suggest that in 1HPT the increased calcium levels, rather than PTH levels, may act as a contributor in the 235 cancer pathogenesis. 
A limit of the present study is related to the lack of reliable data on the vitamin D status during the 10 years prior to diagnosis and even at the time of the study inclusion. Indeed, since our patients were mainly referred to our tertiary care center for osteoporosis and/or fragility fractures, we could not exclude that some of them were taking vitamin D supplements at the time of the diagnosis. This could explain the relatively low prevalence of secondary hyperparathyroidism in our sample. The lack of reliable information on vitamin D status in the patients may be of importance since hypovitaminosis D is associated with an increased risk of neoplasia (35) and in the 1HPT condition the 25OHVitD levels are often reduced (1-4), as suggested even by the present study. Notwithstanding these considerations, the association between the 1HPT condition and the increased occurrence of neoplasia has been confirmed even after adjusting for the vitamin D status.

A further possible explanation of the increased risk of neoplasia associated with 1HPT is that both these conditions could be related to the same genetic predisposition. For example, given the possible effect of vitamin D in influencing the cell proliferation, in particular at the parathyroid glands, it is not possible to exclude that vitamin D status and metabolism and some genetic polymorphic variations in vitamin D metabolism, may have played a role in inducing a predisposition to the development of both parathyroid and neoplastic cells (36). causing substances, chronic inflammation, immunosuppression, obesity, diabetes, radiation, tobacco abuse), have been excluded, there are a number of other factors that could influence the development of cancer (for example the dietary habits and the sun exposure), that have not been assessed. 


\section{Declaration of interest}

259 All authors declare that there is no conflict of interest that could be perceived as prejudicing the impartiality 260 of the research reported.

\section{Funding}

263 This research did not receive any specific grant from any funding agency in the public, commercial or not-

264 for-profit sector. No author has been payed by pharmaceutical companies or other agencies.

\section{Individual contributions}

267 Serena Palmieri: literature search, data collection and analysis, revision of the manuscript

268 Letizia Roggero: literature search, data collection and analysis, drafting of the manuscript

269 Elisa Cairoli: data analysis and interpretation, revision of the manuscript

270 Valentina Morelli: data analysis and interpretation, revision of the manuscript

271 Alfredo Scillitani: study design, data interpretation, revision of the manuscript

272 Iacopo Chiodini: study design, data analysis and interpretation, writing of the manuscript

273 Cristina Eller-Vainicher: study design, data interpretation, revision of the manuscript

275 All authors approved the final version of the manuscript and are accountable for all aspects of the work. The corresponding author (Iacopo Chiodini) had full access to all the data in the study and had final responsibility

277 for the decision to submit for publication. 
278

279

280

281

282

283

284

285

286

287

288

289

290

291

292

293

294

295

296

297

298

299

300

301

302

303

304

305

\section{References}

1. Marcocci C \& Cetani F. Clinical practice. Primary hyperparathyroidism. New England Journal of Medicine 2011365 2389-2397.

2. Fraser WD. Hyperparathyroidism. Lancet 2009374 145-158,

3. Bilezikian JP, Brandi ML, Eastell R, Silverberg SJ, Udelsman R, Marcocci C \& Potts JT Jr. Guidelines for the management of asymptomatic primary hyperparathyroidism: summary statement from the Fourth International Workshop. Journal of Clinical Endocrinology and Metabolism 201499 3561-3569.

4. Silverberg SJ, Clarke BL, Peacock M, Bandeira F, Boutroy S, Cusano NE, Dempster D, Lewiecki EM, Liu JM, Minisola S, Rejnmark L, Silva BC, Walker MD \& Bilezikian JP. Current issues in the presentation of asymptomatic primary hyperparathyroidism: proceedings of the Fourth International Workshop. Journal of Clinical Endocrinology and Metabolism 201499 3580-3594

5. Goswami S \& Ghosh S. Hyperparathyroidism: cancer and mortality. Indian Journal Endocrinology and Metabolism 201216 217-220.

6. Hedback G, Tisell LE, Bengtsson BA, Hedman I \& Oden A. Premature death in patients operated on for primary hyperperathyroidism. World Journal of Surgery 199014 829-836.

7. Whitfield JF, MacManus JP, Youdale T \& Franks DJ. The roles of calcium and cyclic AMP in the stimulatory action of parathyroid hormone on thymic lymphocyte proliferation. Journal of Cellular Physiology 197178 355-368.

8. McCarty MF. Parathyroid hormone may be a cancer promoter - an explanation for the decrease in cancer risk associated with ultraviolet light, calcium, and vitamin D. Medical Hypotheses 200054 475-482.

9. Pettway GJ, Meganck JA, Koh AJ, Keller ET, Goldstein SA \& McCauley LK. Parathyroid hormone mediates bone growth through the regulation of osteoblast proliferation and differentiation. Bone 200842 806-818.

10. Palmér M, Adami HO, Krusemo UB \& Ljunghall S. Increased risk of malignant diseases after surgery for primary hyperparathyroidism - A Nationwide Cohort Study. American Journal of Epidemiology 1988 127 1031-1040.

11. Pickard AL, Gridley G, Mellemkjae L, Johansen C, Kofoed-Enevoldsen A, Cantor KP \& Brinton LA. Hyperparathyroidism and subsequent cancer risk in Denmark. Cancer 200295 1611-1617. 
306

307

308

309

310

12. Øgard CG1, Engholm G, Almdal TP \& Vestergaard H. Increased mortality in patients hospitalised with primary hyperparathyroidism during the period 1977-1993 in Denmark. World Journal of Surgery 2004 28 108-111.

13. Nilsson IL, Zedenius J, Yin L \& Ekbom A. The association between primary hyperparathyroidism and malignancy: Nationwide cohort analysis on cancer incidence after parathyroidectomy. Endocrine Related Cancer 200714 135-140.

14. Palmér M, Adami HO, Bergström R, Akerström G \& Ljunghall S. Mortality after surgery for primary hyperparathyroidism: a follow-up of 441 patients operated on from 1956 to 1979. Surgery 1987102 1-7.

15. Hedbäck G, Odén A \& Tisell LE. Parathyroid adenoma and the risk of death after treatment for primary hyperparathyroidism. Surgery 1995117 134-139.

16.Cinamon U, Levy D \& Marom T. Is Primary Hyperparathyroidism a Risk Factor for Papillary Thyroid Cancer? An Exemplar Study and Literature Review. International Archives of Otorhinolaryngology 2015 $1942-45$.

17. Söreide JA, van Heerden JA, Grant CS, Yau Lo C, Schleck C \& Ilstrup DM. Survival after surgical treatment for primary hyperparathyroidism. Surgery 1997122 1117-1123.

18. Wermers RA, Khosla S, Atkinson EJ, Grant CS, Hodgson SF, O'Fallon WM \& Melton LJ 3rd. Survival after the diagnosis of hyperparathyroidism: a population-based study. American Journal of Medicine 1998 $104115-122$.

19. Eric P, Cohen EP, Krzesinski JM, Launay-Vacher V\& Sprangers B. Onco-Nephrology: Core Curriculum 2015 American Journal of Kidney Disease 201566 869-883.

20. Eller-Vainicher C, Cairoli E, Zhukouskaya VV, Morelli V, Palmieri S, Scillitani A, Beck-Peccoz P \& Chiodini I. Prevalence of subclinical contributors to low bone mineral density and/or fragility fracture. European Journal of Endocrinology 2013169 225-237.

21. Vescini F, Attanasio R, Balestrieri A, Bandeira F, Bonadonna S, Camozzi V, Cassibba S, Cesareo R, Chiodini I, Francucci CM, Gianotti L, Grimaldi, Guglielmi R, Madeo B, Marcocci C, Palermo A, Scillitani A, Vignali E, Rochira V \& Zini M. Italian association of clinical endocrinologists (AME) position statement: drug therapy of osteoporosis. J Endocrinol Invest 201639 807-834. 
333

334

335

336

337

338

339

340

341

342

343

344

345

346

22. Zeichner SB, Stanislaw C \& Meisel JL. Prevention and Screening in Hereditary Breast and Ovarian Cancer. Oncology (Williston Park) 201630 896-904.

23. Klil-Drori AJ, Azoulay L \& Pollak MN. Cancer, obesity, diabetes, and antidiabetic drugs: is the fog clearing? Nature Review Clinical Oncology 2016, epub ahead of print

24. Qian J, Cai M, Gao J, Tang S, Xu L \& Critchley JA. Trends in smoking and quitting in China from 1993 to 2003: National Health Service Survey data. Bulletin of the World Health Organization 201088 769776.

25. Jodkowska A, Tupikowski K, Szymczak J, Bohdanowicz-Pawlak A, Bolanowski M \& BednarekTupikowska G. Interdisciplinary aspects of primary hyperparathyroidism: symptomatology in a series of 100 cases. Advances in Clinical and Experimental Medicine $201625285-293$.

26. Osteoporosis prevention, diagnosis, and therapy. NIH Consensus Statement 200017 1-45.

27. Ferrari S, Bianchi ML, Eisman JA, Foldes AJ, Adami S, Wahl DA, Stepan JJ, de Vernejoul MC \& Kaufman JM; IOF Committee of Scientific Advisors Working Group on Osteoporosis Pathophysiology. Osteoporosis in young adults: pathophysiology, diagnosis, and management. Osteoporososis International $2012232735-2748$.

28. Husten CG. How should we define light or intermittent smoking? Does it matter? Nicotine \& Tobacco Research 200911 111-121.

29. Rossini M, Adami S, Bertoldo F, Diacinti D, Gatti D, Giannini S, Giusti A, Malavolta N, Minisola S, Osella G, Pedrazzoni M, Sinigaglia L, Viapiana O \& Isaia GC. Guidelines for the diagnosis, prevention and management of osteoporosis. Reumatismo 201668 1-39.

30. Eastell R, Arnold A, Brandi ML, Brown EM, D'Amour P, Hanley DA, Rao DS, Rubin MR, Goltzman D, Silverberg SJ, Marx SJ, Peacock M, Mosekilde L, Bouillon R \& Lewiecki EM. Diagnosis of asymptomatic primary hyperparathyroidism: proceedings of the Third International Workshop. Journal of Clinical Endocrinology and Metabolism 200994 340-350.

31. UpToDate calculator. In Calcium Correction in Hypoalbuminemia, version 18.2. Waltham, MA: Wolters Kluwer-Health (available: www.uptodate.com, accessed 2 September 2009), 2010

32. Genant HK, Wu CY, van Kuijk C, Nevitt MC. Vertebral fracture assessment using a semi-quantitative technique. Journal of Bone and Mineral Research 19938 1137-1148. 
361 33.Cairoli E, Eller-Vainicher C, Ulivieri FM, Zhukouskaya VV, Palmieri S, Morelli V, Beck-Peccoz P \&

362 Chiodini I. Factors associated with bisphosphonate treatment failure in postmenopausal women with

363 primary osteoporosis. Osteoporososis International 201425 1401-10.

364 34. Almquist M, Manjer J, Bondeson L \& Bondeson AG. Serum calcium and breast cancer risk: Results

365 from a prospective cohort study of 7847 women. Cancer Causes and Control 200718 595-602.

366 35. Li M, Chen P, Li J, Chu R, Xie D \& Wang H. Review: the impacts of circulating 25-hydroxyvitamin D

367 levels on cancer patient outcomes: a systematic review and meta-analysis. Journal of Clinical

368 Endocrinology and Metabolism 201499 2327-2336.

369 36. Gandini S, Gnagnarella P, Serrano D, Pasquali E \& Raimondi S. Vitamin D receptor polymorphisms and 370 cancer. Advances in Experimental Medicine and Biology 2014 810 69-105. 


\section{$371 \quad$ Legend to Figure 1}

372 Title: Design of the study

373 Footnotes: Exclusion Criteria: 1) the finding of osteoporosis and/or fragility fracture and/or increased

374 calcium and/or PTH levels in the context of investigations for the staging or follow-up of malignancy; 2) the

375 patients' addressing for the need of the prescription of an antifracture drug in the context of aromatase

376 inhibitors therapy; 3 ) the presence of neoplasia in the context of a previously identified familiar and/or

377 hereditary syndrome; 4) the presence of diabetes, obesity, chronic liver diseases or other chronic disorders

378 and/or therapies and/or substances (i.e. glucocorticoids, immunosuppressants, alcohol, external radiation)

379 known to increase the cancer risk; 5) the presence of heavy smoking habit ( $\geq 1$ pack/day)

\section{$381 \quad$ Legend to Figure 2}

382 Title: Percentage of subjects with the occurrence of cancer within 5 years before the study entry among

383 patients with cancer and primary hyperparathyroidism (1HPT) and controls with cancer.

384 The neoplasia occurred within 5 years before the study entry in the $74.3 \%$ of 1 HPT patients with cancer and 385 in the $22.3 \%$ of non-1HPT patients with cancer $(\mathrm{p}<0.0001)$. 
Table 1: Clinical characteristics of the whole sample of subjects included in the study

\begin{tabular}{|c|c|}
\hline & $\begin{array}{l}\text { All patients } \\
(n=1606)\end{array}$ \\
\hline Age (years) & $65.4 \pm 11.0(42-93)$ \\
\hline Gender (females) & $1407(87.6)$ \\
\hline Hypertension & $450(28.0)$ \\
\hline Cardiovascular events ${ }^{1}$ & $110(6.8)$ \\
\hline Dyslipidemia & $233(14.5)$ \\
\hline Osteporosis $^{2}$ & $1375(85.6)$ \\
\hline Osteopenia $^{3}$ & $209(13.0)$ \\
\hline Fragility fractures $^{4}$ & $1103(68.7)$ \\
\hline Smokers & $308(19.2)$ \\
\hline Calcium (mg/dL) & $9.6 \pm 0.6(8.1-13.2)$ \\
\hline Phosporous (mg/dL) & $3.5 \pm 0.6(1.9-4.8)$ \\
\hline Parathyroid hormone (pg/mL) & $34.4 \pm 41.3(9.4-806.0)$ \\
\hline 24-h urine calcium (mg/day) & $196.4 \pm 119.9(60-980.0)$ \\
\hline 25OHVitamin D (ng/mL) & $41.3 \pm 18.9(2.0-133.0)$ \\
\hline All cancers & $214(13.3)$ \\
\hline Breast cancer $^{5}$ & $105(7.5)^{4}$ \\
\hline Prostate cancer $^{6}$ & $7(3.5)^{5}$ \\
\hline Thyroid cancer & 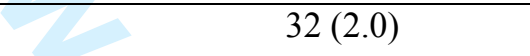 \\
\hline Colon cancer & $17(1.1)$ \\
\hline Uterus cancer & $13(0.9)$ \\
\hline Lung Cancer & $8(0.5)$ \\
\hline Kidney Cancer & $8(0.5)$ \\
\hline Skin cancer & $7(0.4)$ \\
\hline Ovary cancer & $4(0.3)^{4}$ \\
\hline Liver cancer & $4(0.2)$ \\
\hline Pancreas cancer & $3(0.2)$ \\
\hline
\end{tabular}

The results are expressed as mean \pm SD or absolute number, with range or percentage in parentheses.

${ }^{1}$ myocardial infarction, stroke, transient ischemic attack, angina pectoris, pulmonary embolism, intracerebral hemorrhage, peripheral artery disease. ${ }^{2}$ Bone mineral density at any site expressed as T-score below -2.5 and/or presence of fragility fractures. ${ }^{3}$ Bone mineral density at any site expressed as T-score between -1.0 and -2.0. ${ }^{4}$ Clinical fragility fracture spine, ribs, wrist, hip, and proximal humerus and/or morphometric vertebral fractures. ${ }^{5}$ Percentage in female patients. ${ }^{6}$ Percentage in male patients. Smoker: patient who smokes more than 5 cigarettes/day and/or 10 packs/year 
Table 2. Comparison between the clinical and biochemical characteristics and the prevalence of cancer in patients with $1 \mathrm{HPT}$ and without $1 \mathrm{HPT}$

\begin{tabular}{|c|c|c|c|}
\hline & $\begin{array}{l}\text { Patients with 1HPT } \\
(n=163)\end{array}$ & $\begin{array}{l}\text { Patients without 1HPT } \\
\qquad(\mathrm{n}=1443)\end{array}$ & $\mathbf{p}$ \\
\hline Age (years) & $66.5 \pm 12.4(42-90)$ & $65.2 \pm 10.8(42-93)$ & 0.152 \\
\hline Gender (females) & $148(90.8)$ & $1259(87.2)$ & 0.192 \\
\hline Hypertension & $54(33.1)$ & $396(27.4)$ & 0.125 \\
\hline Cardiovascular events $^{1}$ & $15(9.2)$ & $95(6.6)$ & 0.210 \\
\hline Dyslipidemia & $20(12.3)$ & $213(14.8)$ & 0.392 \\
\hline Osteporosis $^{2}$ & $123(75.5)$ & $1252(86.8)$ & 0.875 \\
\hline Osteopenia $^{3}$ & $18(11.0)$ & $191(13.2)$ & 0.875 \\
\hline Fragility fractures $^{4}$ & $83(50.9)$ & $1020(70.7)$ & 0.001 \\
\hline Smokers & $10(6.1)$ & $298(20.7)$ & 0.001 \\
\hline Calcium (mg/dL) & $10.7 \pm 0.6(10-13.2)$ & $9.5 \pm 0.4(8.1-10.4)$ & 0.001 \\
\hline Phosporous (mg/dL) & $2.7 \pm 0.5(1.9-4.3)$ & $3.6 \pm 0.5(2.7-4.8)$ & 0.001 \\
\hline Parathyroid hormone $(\mathrm{pg} / \mathrm{mL})$ & $124.2 \pm 85.5(30.0-806.0)$ & $24.3 \pm 8.0(9.4-134.0)$ & 0.001 \\
\hline 24-h urine calcium (mg/day) & $291.1 \pm 157.5(90.0-980.0)$ & $185.5 \pm 109.8(60.0-975.0)$ & 0.001 \\
\hline 25OHVitamin D (ng/mL) & $36.2 \pm 17.7(4.0-88.0)$ & $41.8 \pm 18.9(4.0-133.0)$ & 0.001 \\
\hline All cancers & $35(21.5)$ & $179(12.4)$ & 0.001 \\
\hline Breast cancer $^{5}$ & $18(12.2)$ & $87(6.9)$ & 0.021 \\
\hline Prostate cancer $^{6}$ & $0(0.0)$ & $7(3.8)$ & 1.000 \\
\hline Thyroid cancer & $6(3.7)$ & $26(1.8)$ & 0.104 \\
\hline Colon cancer & $1(0.6)$ & $12(0.8)$ & 1.000 \\
\hline Uterus cancer & $1(0.7)$ & $12(1.0)$ & 1.000 \\
\hline Lung Cancer & $2(1.2)$ & $6(0.4)$ & 0.191 \\
\hline Kidney Cancer & $4(2.5)$ & $4(0.3)$ & 0.005 \\
\hline Skin cancer & $3(1.8)$ & $4(0.3)$ & 0.026 \\
\hline Ovary cancer & $1(0.7)$ & $3(0.2)$ & 0.359 \\
\hline Liver cancer & $0(0.0)$ & $4(0.3)$ & 1.000 \\
\hline Pancreas cancer & $1(0.6)$ & $2(0.1)$ & 0.275 \\
\hline
\end{tabular}

The results are expressed as mean $\pm \mathrm{SD}$ or absolute number, with range or percentage in parentheses.

${ }^{1}$ myocardial infarction, stroke, transient ischemic attack, angina pectoris, pulmonary embolism, intracerebral hemorrhage, peripheral artery disease. ${ }^{2}$ Bone mineral density at any site expressed as T-score below -2.5 and/or presence of fragility fractures. ${ }^{3}$ Bone mineral density at any site expressed as T-score between -1.0 and -2.0. ${ }^{4}$ Clinical fragility fracture spine, ribs, wrist, hip, and proximal humerus and/or morphometric vertebral fractures. ${ }^{5}$ Percentage in female patients. ${ }^{6}$ Percentage in male patients. Smoker: patient who smokes more than 5 cigarettes/day and/or 10 packs/year. 
Table 3. Association between the presence of all cancer (panel A), skin cancer (panel B), kidney cancer (panel C) and breast cancer (panel D) with the presence of 1HPT, after adjusting for hypovitaminosis, smoking habit, gender and age.

\begin{tabular}{|c|c|c|c|}
\hline & Odds ratio & $P$ value & $95 \% \mathrm{CI}$ \\
\hline \multicolumn{4}{|l|}{ Panel A: All Cancer } \\
\hline Age (1 year increase) & 1.029 & 0.000 & $(1.015-1.042)$ \\
\hline Female gender & 1.837 & 0.031 & $(1.058-3.191)$ \\
\hline Hypovitaminosis D (yes) & 1.354 & 0.082 & $(0.963-1.903)$ \\
\hline Smoker (yes) & 1.066 & 0.742 & $(0.729-1.558)$ \\
\hline 1HPT diagnosis (yes) & 1.928 & 0.002 & $(1.270-2.929)$ \\
\hline \multicolumn{4}{|l|}{ Panel B: Skin Cancer } \\
\hline Age (1 year increase) & 1.013 & 0.682 & $(0.952-1.037)$ \\
\hline Female gender & 1.387 & 0.765 & $(0.162-11.904)$ \\
\hline Hypovitaminosis D (yes) & 3.368 & 0.270 & $(0.389-21.192)$ \\
\hline Smoker (yes) & 1.012 & 0.991 & $(0.116-8.849)$ \\
\hline 1HPT diagnosis (yes) & 8.236 & 0.008 & $(1.717-39.509)$ \\
\hline \multicolumn{4}{|l|}{ Panel C: kidney cancer } \\
\hline Age (1 year increase) & 1.025 & 0.429 & $(0.964-1.091)$ \\
\hline Hypovitaminosis D (yes) & 1.563 & 0.592 & $(0.306-7.981)$ \\
\hline Smoker (yes) & 1.035 & 0.975 & $(0.120-8.928)$ \\
\hline 1HPT diagnosis (yes) & 9.184 & 0.003 & $(2.155-38.825)$ \\
\hline \multicolumn{4}{|l|}{ Panel D: Breast cancer } \\
\hline Age (1 year increase) & 1.024 & 0.013 & $(1.005-1.043)$ \\
\hline Hypovitaminosis D (yes) & 1.432 & 0.139 & $(0.890-2.306)$ \\
\hline Smoker (yes) & 1.153 & 0.590 & $(0.688-1.932)$ \\
\hline 1HPT diagnosis (yes) & 1.930 & 0.020 & $(1.112-3.352)$ \\
\hline
\end{tabular}

1HPT: primary hyperparathyroidism. Smoker: patient who smokes more than 5 cigarettes/day and/or 10 packs/year. Hypovitaminosis D: 25-hydroxy-vitamin D levels below $30 \mathrm{ng} / \mathrm{mL}$ 


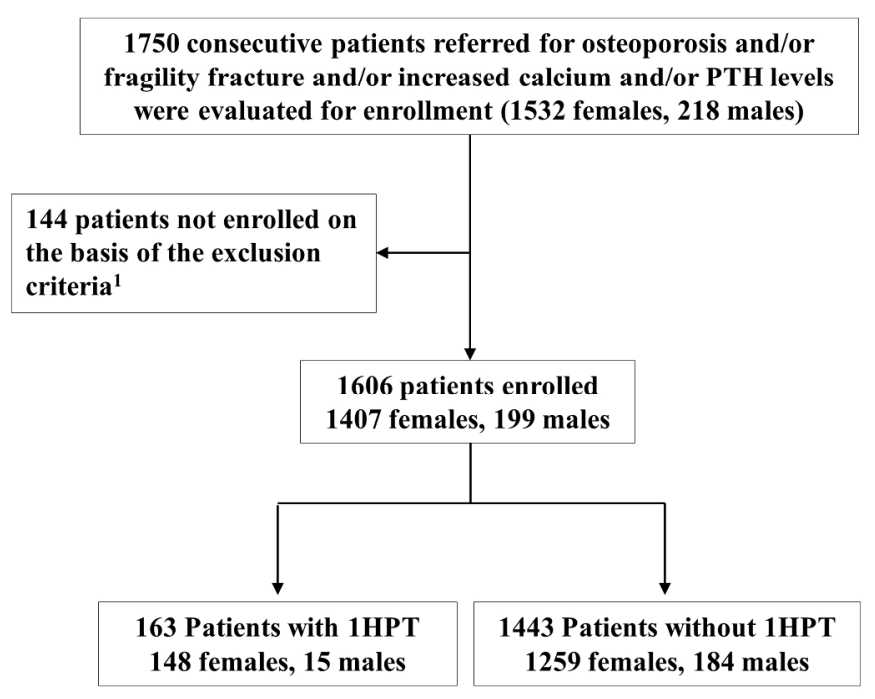

Figure 1: Design of the study

Exclusion Criteria: 1) the finding of osteoporosis and/or fragility fracture and/or increased calcium and/or PTH levels in the context of investigations for the staging or follow-up of malignancy; 2 ) the patients' addressing for the need of the prescription of an antifracture drug in the context of aromatase inhibitors therapy; 3 ) the presence of neoplasia in the context of a previously identified familiar and/or hereditary syndrome; 4) the presence of diabetes, obesity, chronic liver diseases or other chronic disorders and/or therapies and/or substances (i.e. glucocorticoids, immunosuppressants, alcohol, external radiation) known to increase the cancer risk; 5) the presence of heavy smoking habit ( $\geq 1$ pack/day)

$190 \times 274 \mathrm{~mm}(284 \times 284 \mathrm{DPI})$ 


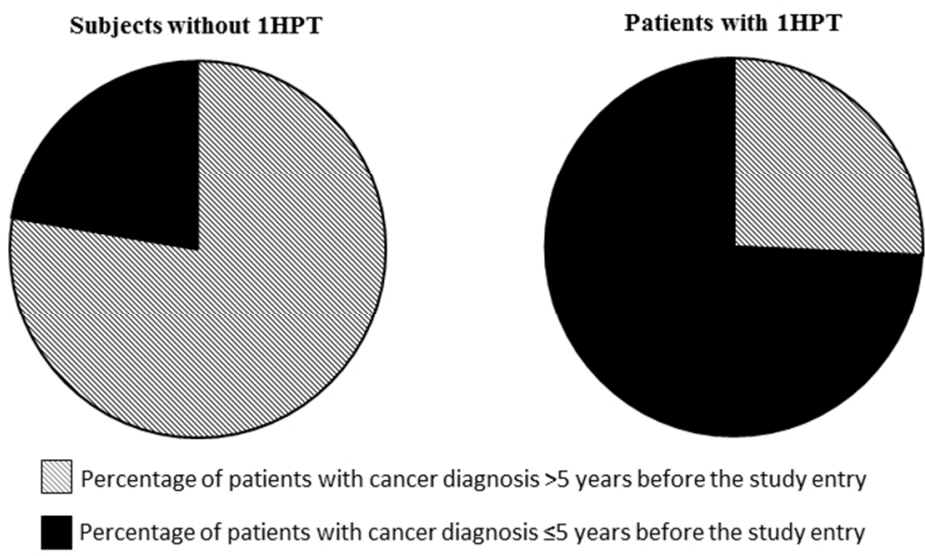

Figure 2: Percentage of subjects with the occurrence of cancer within 5 years before the study entry among patients with cancer and primary hyperparathyroidism (1HPT) and controls with cancer.

The neoplasia occurred within 5 years before the study entry in the $74.3 \%$ of 1 HPT patients with cancer and in the $22.3 \%$ of non-1HPT patients with cancer $(p<0.0001)$.

$254 \times 190 \mathrm{~mm}(96 \times 96 \mathrm{DPI})$ 